\title{
L'Africa in cortile: la colonia nelle storie levantine di Fausta Cialente
}

\section{Giuliana Minghelli}

Cortile a Cleopatra, titolo del secondo romanzo della Cialente, invita sin dall'inizio il lettore a tracciare o rintracciare quella che Edward Said, in Orientalism, definisce una geografia immaginaria. Anche dopo aver saputo che Cleopatra è un sobborgo di Alessandria di Egitto, il titolo si sottrae ad una esatta figurazione spaziale. Questo soprattutto a causa dell'uso della preposizione $a$ che, significando contemporaneamente l'in e l'ad latino, accosta due spazi senza predicarne apertamente la relazione: lo spazio contenuto del cortile e (vicino, opposto, contiguo) un sobborgo, Cleopatra, anticamera alla città e alla campagna. La domanda che sorge è la seguente: è uno spazio semplicemente contenuto dall'altro o è il cortile anfiteatro, privilegiato punto di osservazione, all'entroterra? Quale funzione svolgano questi luoghi nella costruzione dell'identità dei personaggi, quale relazione li leghi allo spazio della colonia ed infine come la geografia spicciola, quotidiana da essi tracciata narrativizzi l'instabilità che lega Africa ed Europa, sono le domande che guideranno questa riflessione.

Ma prima di procedere a perlustrare il cortile e i suoi dintorni seguendo i vagabondaggi del protagonista Marco, s'impone una breve considerazione sulla posizione occupata dalla narratrice che, a sua volta, scrive in Africa e sull'Africa. Edward Said a questo proposito osserva:

Everyone who writes about the Orient must locate himself vis-à-vis the Orient; translated into his text, this location includes the kind of narrative voice he adopts, the type of structure he builds, the kinds of images, themes, motifs that circulate in his texts - all of which add up to deliberate ways of addressing the reader, containing the Orient, and finally, representing it or speaking in its behalf. (20)

Secondo Said presupposto necessario alla rappresentazione è l'esteriorità che permette di contenere l'Oriente, di farlo parlare. "The orientalist, poet or scholar, makes the orient speak ... [by occupying a position] outside the orient, both as an existential and as a moral fact" (21). Che posizione occupa la Cialente in Cortile a Cleopatra, qual è l'oggetto della sua rappresentazione, per chi parla? Diversi sono gli oggetti rappresentati: Marco, l'italiano esule in Africa; la colonia, costituita dagli abitanti del cortile; l'Egitto esotico. Questa pluralità di oggetti suggerisce una pluralità di posizioni soggettive che nega un punto di vista privilegiato. L'Europa - la cui identita, secondo l'analisi di Said, si costituisce nel 
contrapporsi all'alterità dell'oriente —, lungi dal costituire una soggettività sovrana, sopravvive solo come memoria e possibilità. La Cialente, scrivendo vis à vis l'Egitto presente e misterioso, l'Europa famigliare e lontana, la colonia amata e odiata, finisce per occupare un luogo al di fuori di sé e per scrivere, vis à vis se stessa, un'identità displaced e riinscritta sulla carta geografica. Quell'Oriente che ha aiutato a definire l'Europa, nella Cialente, offre un palcoscenico per la sua dissoluzione. È su questo palcoscenico che ora ci avventuriamo.

Seduta sul ramo basso del fico la scimmia sorvegliava Marco che dormiva lì sotto sdraiato all'ombra festosa e ondeggiante delle foglie; dormiva con la bocca aperta . . . . La terra del cortile era dura. Le piccole case intorno, basse e a pianoterra, decrepite e miserabili, avevan tutte le loro storte persiane chiuse . . . . Voci di fanciulle nell'aria, d'improvviso . . . : erano voci fresche che andavano e venivano da un muro all'altro del cortile quadrato e come fili tessevano per il suo sonno un cielo di pace. Il mare si quieto dietro la casa. (19-20)

Conosciamo il mondo di Cortile a Cleopatra attraverso gli occhi di Marco, che, sdraiato al centro del cortile all'ombra del fico, costituisce il perno su cui ruotano le vite degli altri personaggi. Marco è figlio di Crissanti, una donna greca che vive nella casa sul mare, e di Alessandro, il pittore/decoratore italiano che, dopo essersi sposato in Egitto, ha abbandonato la moglie portando con sé in Italia il bambino nato nel frattempo. Alla morte del padre, Marco, senz'arte né parte, senza ambizioni né voglia di lavorare, salpa per l'Egitto alla ricerca della madre. All'inizio del romanzo lo troviamo nel cortile addormentato sotto il fico, a bocca aperta, quasi in oziosa attesa dei frutti che, ancora piccoli e bianchi, una volta maturati, gli cadranno in bocca. Come questi fichi, le donne del cortile - Haiganùsh, la ragazza armena, Dinah, la figlia del ricco ebreo Abramino, Eva, madre di Dinah, Kikì, la ragazza mulatta - cadranno vittime del suo amore pigro e distaccato.

Sebbene Marco sieda al centro del cortile, lo fa come un ozioso osservatore che si è trovato per caso nel cuore della piccola comunità levantina. Spinto dall'amore per Dinah a farne parte, finisce per vivere nella società del cortile in uno stato di assenza, come in sogno, e reprimendo a malapena il desiderio di fuggire. Accetta un lavoro come manovale e subito cerca delle scuse per sottrarsene, si fidanza con Dinah e presto ne rifiuta l'amore inquinato da aspirazioni piccolo borghesi, accetta di lavorare nella soffocante bottega di pelli di Abramino e, in un tentativo di fuga, seduce la bella Eva, un atto che precipita la tragedia finale e la sua fuga. Questa altalena esistenziale tra il centro e i margini coincide con un'altalena tra l'interno e l'esterno del cortile, un'altalena tra lo spazio della colonia e l'Africa, tra identità e differenza.

All'interno del cortile gli spazi sono rigidamente assegnati e rinchiudono i vari personaggi, se si vuole, le varie etnie, come in una gabbia. Così nella scena iniziale l'armena Haiganùsh e Dinah, l'amica ebrea italiana, appaiono sui balconi come nella cornice di un quadro: "In piedi sul balconcino cadente, bianca e scalza 
contro il muro d'un verde azzurro e tenero come quello di un affresco . . . Dinah piegava la testa sulla spalla" (22). Quando il marito della sarta, il vecchio greco Spiro Triandafilu, compare alla finestra svegliato dalle grida "si muove nel balconcino come dentro una gabbia, agitando le mani, la testa, le spalle" (28). La bella Eva, che spesso Marco segue con lo sguardo, è a malapena contenuta in questa povera cornice o così pensa Marco: “Abramino è pazzo di tenersi per moglie quella figura dipinta e lussuosa, e volere che rimanga inquadrata nelle persiane sgangherate e storte d'una povera finestra aperta in un muro scalcinato ..." (59). Mentre la casa della madre e il cortile sono per Marco delle gabbie - da cui egli, come la sua scimmia, fugge dalla finestra - il paesaggio esterno offre vasti orizzonti al suo desiderio di vagabondare.

Invece di uscire dalla porta, scavalco la finestra e scese sul terrapieno a destra della casa. Il terreno era tutto sparso di rifiuti e immondizie, vetri di bottiglie, scarpe rotte . . reticolati divelti dai pali e contorti, resti degli accampamenti di guerra . . . Si fece ombra con le mani sugli occhi e guardò a est, verso Mustàfa, le gialle caserme inglesi, il minareto della moschea di Sidi Gaber e poi verso terra la stazione di Cleopatra. Le case intorno al cortile erano le ultime sulla spiaggia, sull'orlo della scarpata, sole in mezzo all'ondulazione dei terrapieni deserti; piccole e basse, pitturate all'esterno di un rosa stinto e scalcinato, animate dallo svolazzare dei bucati tesi a festoni in alto sui terrazzi. La casa di Abramino, pellicciaio e proprietario, era la meno rovinata, in quella di faccia abitavano i due inquilini, la sarta [greca] e il calzolaio [armeno]. In fondo, sul mare, la piccola casa di sua madre. Verso terra, sul viottolo, c'era lo steccato con il cancello che non si poteva chiudere . . . . In mezzo si vedeva sorgere la testa verde del fico. (34-35)

In questo panorama la geografia diviene apertamente cifra allegorica del potere e della storia. La linea dell'orizzonte che abbraccia il cortile è disegnata dalle caserme inglesi (legge e ordine senza di cui, come esclama la nonna di Daniela in Ballata levantina, "Chissà dove saremmo!"), dalla moschea e dal minareto arabi, dalla stazione di Cleopatra - che individua questo sobborgo come un luogo di arrivi e partenze - e, alle spalle di tutto, dal mare, la linea di confine con l'Europa, alla cui vista Marco si perde a fantasticare dell'Italia e del padre. ${ }^{1}$ Le coordinate geografiche della colonia sono così tracciate e il cortile ne rappresenta la materializzazione metaforica.

Le case raccolte intorno al cortile, come le etnie greca, armena, italiana, ebrea, francese, sono unite nella loro diversità a formare la comunità levantina. Queste case sono "le ultime sulla spiaggia, sull'orlo della scarpata, sole in mezzo all'ondulazione dei terrapieni deserti" (34). Distaccate dai rumorosi quartieri arabi, sembrano essere state portate dal mare, e il mare costantemente minaccia di reclamarsele. Vivere tra l'Africa e l'Europa significa abitare un terreno in un costante stato di dissoluzione, sparso di cocci e vetri di bottiglie, una terra di nessuno che raccoglie i frammenti di diverse civiltà. I levantini, forse perché consci di questo pericolo, scelgono di vivere ingabbiati in case che offrono un effimero senso di identità e appartenenza, si illudono di occupare un punto privilegiato di cono- 
scenza e osservazione sul mondo indigeno, arrancano nell'inseguimento di prestigio sociale e fortuna. L'irrequietezza di Marco ("mezzoitaliano, scamiciato, vagabondo") mette a nudo questo gretto senso di appartenenza. Anche se la colonia levantina ha fatto vangelo del motto di Disraeli "The East is a career", il senso di identità dei suoi membri, indipendentemente da razza o religione, ha ben poco a che spartire col "royal English subject".

Vale la pena ricordare a questo punto che la parola colonia trova il suo etimo nel termine colono: colui che coltiva la terra per conto d'altri, una terra che non gli appartiene. La colonia levantina lungi dall'esprimere una sovranità sul territorio esprime piuttosto questo displacement, una marginalità definita da un centro che si trova altrove, nel dominio imperialistico inglese che ne garantisce l'esistenza o nella popolazione indigena unica vera padrona della terra. Nonostante i suoi sensi di casta (divisioni dettate più da ragioni economiche che etniche), la colonia è uno spazio dove le identità e le differenze sono in uno stato di perenne flusso, una zona di costanti negoziazioni, etniche, politiche, sessuali, linguistiche. Questo carattere ibrido è direttamente indicato nella parola "levantino", un precipitato che include egiziano, italiano, greco, ebreo apolide e meticcio. In "What is a $\mathrm{Na}$ tion?" Ernest Renan riflette sulla precarietà dell'identità coloniale se messa a confronto con "l'essenza" che contraddistingue una nazione e osserva:

If you take a city such as Salonika or Smyrna, you will find there five or six communities each of which has its own memories and which have almost nothing in common. Yet the essence of a nation is that all individuals have many things in common, and also that they have forgotten many things. (11)

Ma, paradossalmente, proprio il rischio di dimenticare unisce i disparati abitanti della colonia, dando loro, se non un'essenza, un senso di comunità che nulla ha da invidiare all'abitante di una nazione. La parola "levantino", sebbene estremamente volatile ed instabile, finisce cosi per tracciare una nuova geografia rinchiusa tra l'impero inglese e il continente africano, due alterità che non possono essere contenute ma che letteralmente e simbolicamente contengono.

Haiganùsh durante una lite con Marco esprime inconsciamente una caratteristica che vale a descrivere la condizione ibrida degli abitanti della colonia:

"Italiano che non sei e nemmeno greco, religione non hai, in nessuna chiesa puoi entrare, sei meno d'un bastardo, con tutto il rispetto che devo a quella povera donna di tua madre". (26, enfasi mia)

Come Marco, i levantini sono, anche se solo metaforicamente, figli illegittimi, nati al di fuori di una nazione, frutto di incroci tra diversi gruppi etnici. Sono metaforicamente senza padre, perché è il padre a porti all'interno di una genealogia, a sancire la tua appartenenza ad una terra (patria). Non sorprende quindi che in Ballata levantina, i levantini facciano appello alla forza redentrice del padre putativo, Mussolini, che ha il potere di redimere i figli dispersi, un padre che tutte le altre etnie, persino gli inglesi, invidiano agli italiani. 
Ma i personaggi della Cialente, Marco, Haiganùsh, Kikì ed eventualmente Dinah e anche Daniela in Ballata levantina, piuttosto che essere bastardi, sono orfani di padre o di madre, di padre e di madre e quindi della "madrepatria". Questa loro condizione, al di là del suo aspetto letterale, punta ad un'assenza (orfano significa essere privo) intrinseca al soggetto della colonia. Teorizzando questa assenza, Deleuze e Guattari scrivono in Anti-Oedipus:

Desire does not lack anything; it does not lack its object. It is, rather, the subject that is lacking in desire, or desire that lacks a fixed subject; there is no fixed subject except by repression.... Desire is machine, the object of desire also a connected machine, so that the product is lifted from the process of producing, and something detaches itself from producing to product and gives a leftover to the vagabond, nomad subject. (26)

Marco, il soggetto coloniale frammentato, "lacks in desire". Nel momento in cui incontra l'oggetto del suo desiderio - nel momento in cui ritrova sua madre, si fidanza con Dinah o entra nella società levantina del denaro e lavoro - è messo a confronto con la sua assenza, il suo non essere dove è il desiderio. Quest'assenza - non ascrivibile all'altro ma insita, inseparabile dal soggetto - è vissuta come un letterale vagabondaggio, un nomadismo sui carri dei sudanesi in viaggio verso il cuore dell'Africa. Questi vagabondaggi di Marco, il soggetto occidentale perso nel suo stesso labirinto, ironicamente hanno luogo attorno al 1936, anno in cui ha inizio la campagna etiopica, tarda allucinazione mussoliniana di un impero coloniale, ultima spiaggia per rappresentare, contenere, dominare l'altro.

Pur lasciando in ombra le più immediate coordinate storiche, Cortile a Cleopatra pud essere pienamente inteso come un romanzo di fuga ed esilio solo se messo in relazione con l'orizzonte storico del fascismo. ${ }^{2}$ Solo in questa prospettiva acquista profondità e significato un personaggio senza futuro, senza casa, senza patria, come Marco; solo in questa prospettiva il suo oblomovismo, o nell'espressione di Emilio Cecchi il suo "parassitismo aureolato di poesia", acquistano un valore non mitico, bensì storico ed esistenziale. ${ }^{3} \mathrm{D}$ 'altra parte questo soggetto, che si sottrae ad anagrafi, trame esistenziali e nazionali, continua, a sessant'anni di distanza, a interrogare la natura dei confini che rinchiudono un individuo in una società, nazione o razza. Emilio Cecchi nell'introduzione del 1953 al romanzo ignora questo aspetto, sottolineando invece come il personaggio di Marco sia impensabile nella società del dopoguerra, dove

incapperebbe nelle maglie di regolamenti e nelle internazionali coercizioni "for displaced people". Dovrebbe insomma prima di tutto cominciare a mettersi in regola con la etnografia, la geografia, e specialmente con la politica. (6)

Ma cosa significa mettersi in regola con etnografia, geografia e politica? L'anticonformismo di Marco mette in discussione la natura di queste categorie tassonomiche: vivendo ai margini, nelle fratture tra etnografie, geografie, e assetti politici diversi, questo personaggio drammatizza il carattere discorsivo e fantasmati- 
co di ogni ordine, vecchio e nuovo, del mondo. Per Marco, come per Geoffrey Bennington, "Frontiers are articulations, boundaries are, constitutively, crossed or transgressed" (Bennington 121).

Non sorprende quindi che la colonia da luogo di conquista, sia divenuta nella Cialente uno spazio virtuale in cui si vive in presenza di una assenza. C'è una frase che troviamo sulle labbra di Daniela bambina, la protagonista di Ballata levantina, che getta una luce inaspettata sul carattere e significato di quest'assenza. La sua osservazione è legata alla scoperta degli eunuchi, personaggi grassocci dai fianchi larghi che sorvegliano la villa accanto a casa sua:

Sentivo dire intorno a me: siriani, armeni, maroniti, e credevo che anche gli eunuchi fossero $i$ componenti di una nazione o di una setta religiosa, adatti, chissà mai per quale ragione, alla sorveglianza degli harem; e poiché la moda degli harem andava tramontando, adesso stavano sui cancelli con l'aria oziosa dei disoccupati. $(65-66)$

Quest'associazione nella sua assurdità contiene un'illuminazione. Le varie etnie levantine, separate dalla madrepatria e ormai legate da stretti vincoli all'Egitto (all'altro), sono allo stesso tempo altri delle popolazioni indigene e dei loro lontani compatrioti. Come soggetti che hanno un accesso al mercato ma sono privati di un pieno status politico sono castrati. Questa loro condizione, simile alla condizione di Zambinella analizzata da Roland Barthes in $S / Z$, segna il collasso di un'economia basata su un semplice binarismo tra colonizzatore e colonizzato (inglese/egiziano), soggetto conoscente e oggetto della sua conoscenza, interno ed esterno, Africa ed Europa. ${ }^{4}$ Quindi ritornando alle considerazioni iniziali possiamo dire che il cortile a Cleopatra ha allo stesso tempo una vista sull'entroterra africano e a questo appartiene. Il levantino fa parlare e rappresenta l'Africa, ma soprattutto l'Africa parla attraverso di lui/lei, come testimoniano i numerosi prestiti che arricchiscono e storpiano lingue natie in parte dimenticate. ${ }^{5}$

Questa posizione non va letta come puramente negativa. Certamente non i vagabondaggi di Marco, che possono essere interpretati come una fuga senza frutto; ma la rappresentazione fattane dalla Cialente inaugura la possibilità, per il soggetto occidentale, di occupare una diversa posizione, una posizione che Trinh Minh-Ha descrive eloquentemente parlando delle donne post-coloniali in questi termini:

The moment the insider steps out from the inside she's no longer a mere insider. She necessarily looks in from the outside while also looking out from the inside. Not quite the same, not quite the other, she stands in that undetermined threshold place where she constantly drifts in and out. ${ }^{6}$ ("Not You" 76)

Trasgredendo, con la rappresentazione dello spazio della colonia e della variegata comunità levantina, l'opposizione tra interno ed esterno, Fausta Cialente occupa e crea una nuova posizione; quella dell' "inappropriate other or same" che scrive abbandonandosi contemporaneamente a due gesti: 
that of affirming 'I am like you' while persisting in her difference and that of reminding 'I am different' while unsettling every definition of otherness arrived at. (Trinh, "Not You" 77)

Per questo soggetto che vive sulla soglia, la geografia della colonia levantina finisce per sovrapporsi, senza tuttavia coincidere, con la geografia indigena e tracciare uno spazio e un orizzonte virtuali. Opposta allo spazio chiuso del cortile, la strada costituisce la cerniera tra questi orizzonti e, allo stesso tempo, si impone come simbolo di non-appartenenza. Marco, nella sua fuga dal cortile, non trova rifugio nell'Egitto dei fellah, ma semplicemente vaga "in cerca di una strada, una buona strada come ce ne sono in Italia, di quelle che quando s'incominciano non si ha più voglia di lasciarle né di tornare indietro" ( 80$)$, una strada che offra vedute fantastiche dell'Europa al di là del mare e dell'Africa che si nasconde dietro l'orizzonte afoso. Nondimeno gli oziosi vagabondaggi di Marco finiscono per ricondurlo a spazi chiusi e soffocanti come quelli del cortile.

Era arrivato in mezzo al quartiere indigeno .... (Le case basse di fango o di pietre sconnesse fanno della piccola valle di Sidi Gaber un miserabile mucchio che sembra, la notte, l'avanzo di un terremoto) ... . Egli avrebbe voluto affrettare il passo. uscire di là dov'era capitato per distrazione, ma i vicoli erano incastrati gli uni negli altri come in un labirinto, ogni tanto batteva contro un muro e doveva tornare indietro, cercare l'uscita del villaggio buio e accidentato . . . . (137-38)

Emilio Cecchi, nella sua introduzione, nota giustamente come le "pause spaziali scandiscono il corso del tempo e il maturarsi del dramma" (7), e così facendo attira l'attenzione al mutuo e complesso intrecciarsi di spazio e tempo nel romanzo. Il tentativo di Marco di sottrarsi a una geografia obbligata si traduce in un movimento narrativo, cioè temporale, in una fuga dall'incastro della trama, alle "ipotesi di destino" avanzate da vari personaggi. Così facendo egli rimane sulla soglia: la soglia della casa di Dinah, della bottega di Abramino, del cortile, dell'Italia, e infine anche dell'Africa. Infatti, persino la sua fuga da Cleopatra verso l'alto Egitto è una fuga che ha come meta una soglia: "Si vede già seduto sulla soglia delle loro [degli indigeni] piccole case di fango a masticare la canna da zucchero dolce e succosa" (242). Sebbene Marco alla fine romanticizzi la sua fuga dal cortile ("ora va lontano, nell' interno, a vivere piuttosto con gli indigeni che con i levantini" [242]), il suo viaggio va da un interno ad un altro, rispetto al quale egli si situa comunque come un "outsider", "the inappropriate other", ma anche "same", perché, metaforico o letterale che sia, condivide il nomadismo di tutti i soggetti coloniali.

Nella sua estraneità a tutti i mondi rappresentati — all'occidente dell'etica del lavoro, del progresso e della conquista e all'oriente della schiavitù e del sudore quotidiani del fellah - Marco può essere facilmente letto come il simbolo dell'artista, un simbolo reso con spietata crudezza e simpatia dalla Cialente. Cortile a Cleopatra, in questa prospettiva, diviene la rappresentazione dei tempi e 
luoghi della necessaria e tuttavia egoistica ribellione dell'artista, una posizione impossibile da sostenere, un non-luogo, un'utopia, che pur denunciando il presente, non annuncia necessariamente tempi migliori.

Ma Marco non è un artista. Non riuscendo a rappresentare gli spazi della sua esperienza, egli è infine inghiottito dalla geografia da lui stesso tracciata.

Marco cercò di scrivere con una matita qualche parola sulla carta unta e spiegazzata del pane: voleva esprimere di quei datteri, di quel cielo, di tutto quel mistero, ma non gli riuscì. (156)

Dal canto suo, la Cialente, inabitando lo spazio preciso seppur instabile della soglia, può ripensare l'Africa, così presente ai sensi e misteriosa tanto da rischiare di essere ridotta a un cliché, e l'Europa, persa e tuttavia presente casa della memoria. Sulla soglia, richiusa tra questi orizzonti, ha luogo la scrittura della geografia immaginaria che coincide con la scrittura del soggetto nomade ai confini di se stesso.

Come suggerito in precedenza, la virtualità del soggetto coloniale non è legata unicamente allo spazio ma anche al tempo: l'esilio dall'Italia coincide con l'esilio dal "vergognoso periodo fascista". "Le piace il nostro tempo?" chiedeva alla Cialente Sandra Petrignani in una intervista del 1983. "No" - rispondeva la scrittrice - "Ma come si potrebbe preferirne un altro? Non possiamo inventarcelo" (89). Nonostante questa affermazione, la Cialente, in Cortile a Cleopatra come anche in Ballata levantina, ha finito per metter in pratica l'epigrafe apposta da Stefano Benni a Baol: "Se i tempi non chiedono la tua parte migliore/ Inventa altri tempi", 7 un imperativo che la scrittrice ha adeguato al suo gusto realista, inventando con le sue storie invece di altri tempi altre geografie - due cose che, forse, in fondo, si equivalgono.

\section{University of Colorado-Boulder}

\section{NOTE}

1 Il Mediterraneo costituisce un punto di unione, un confine che riavvicina le varie nazioni ed etnie in una grande comunità, contenuta nel significato della parola levantino. Emest Renan, storico francese dell'Ottocento, nel suo saggio "What is a Nation?" sottolinea il carattere particolare di questo confine quando scrive: "It is indisputable that the mountains separate, but the rivers tend rather to unify" (18).

2 Giulio Ferroni, in occasione della recente scomparsa della scrittrice, ha così tracciato le coordinate storiche di Cortile a Cleopatra: "Negli anni Trenta, questo romanzo . . . offri l'immagine di un mondo libero e vitale, lontanissimo dall'orizzonte prevalentemente cupo, 'indifferente' o percorso da vibratili umori, della cultura di quegli anni, oltre che dal clima plumbeo dell'Italia fascista" (4). Nella scarsa letteratura critica sulla Cialente vale la pena ricordare, anche se solo per dissentime, linterpretazione sociologica di Anna Nozzoli che sottrae Cortile a Cleopatra a ogni contestualizzazione storica, sottolineandone piuttosto il presunto carattere mitico e "la simbologia esistenziale". L'unico articolo in lingua inglese, un'utile panoramica dell'intera opera cialentiana, è quello di Paola Malpezzi Price.

3 Vedi l'introduzione di Emilio Cecchi alla seconda edizione del romanzo uscita nel 1952 
per i caratteri di Sansoni e quindi riprodotta nell'edizione Mondadori del 1973, 5-10.

4 Barthes, in particolare "The Three Points of Entry" 214-16.

5 Nell'avvertenza che la Cialente ha fatto precedere all'edizione del 1973, si legge a proposito dell'ibrida lingua coloniale: "pur avendo scritto in un italiano corretto, mi sembrava d'esser riuscita a proporre un insolito linguaggio popolare . . . : l'italiano corrotto degli italiani, il francese ancor più devastato dei greci, degli armeni, degli ebrei, e l'influenza dell'arabo su tutti quanti, lingua spiritosa, vivace e colorita, quindi saporitissima" (15).

6 La riflessione teorica di Trinh Minh-Ha sulla condizione del postcolonial gendered subject (sviluppata piu a fondo in testi come Woman and When the Moon) e strettamente legata alla sua attività cinematografica e per questa ragione riveste un particolare interesse per lo studio della rappresentazione dello spazio in letteratura.

7 Benni, "Baolian" 2.16-17.

\section{OPERE CITATE}

Barthes, Roland. SIZ. New York: Hill and Wang, 1992.

Benni, Stefano. Baol. Feltrinelli: Milano, 1993.

Bennington, Geoffrey. "Postal Politics and the Institution of the Nation". Bhabha 121-37.

Bhabha, Homi, ed. Nation and Narration. New York: Routledge, 1990.

Cialente, Fausta. Cortile a Cleopatra. Milano: Mondadori, 1973.

. Ballata levantina. Milano: Feltrinelli, 1961.

Deleuze, Gilles, and Felix Guattari. Anti-Oedipus: Capitalism and Schizofrenia. New York: Viking Press, 1977.

Ferroni, Giulio. 'La libera passione di Fausta Cialente". L'Unità (13 Marzo 1994): 4.

Malpezzi-Price, Paola. "Autobiography, Art, and History in Fausta Cialente's Fiction". Contemporary Women Writers in Italy. Ed. Santo Aricò. Amherst: U of Massachusetts P, 1990. 109-22.

Nozzoli, Anna. "Fausta Cialente: Testimonianza storica e tipologia femminile". Tabù e Coscienza: La condizione femminile nella letteratura italiana del Novecento. Firenze: La Nuova Italia, 1978. 113-27.

Petrignani, Sandra. "Fausta Cialente: Straniera dappertutto". Le signore della scrittura; interviste. Milano: La Tartaruga, 1984. 83-89.

Renan, Ernst. "What is a Nation?". Bhabha 8-22.

Said, Edward. Orientalism. New York: Vintage Books, 1978.

Trinh, T. Minh-Ha. "Not You/Like You: Postcolonial Women and the Interlocking Questions of Identity and Difference". Inscriptions 3-4 (1988): 71-77.

When the Moon Waxes Red: Representation, Gender, and Cultural Politics. New York: Routledge, 1991. ana UP, 1989.

Woman, Native, Other: Writing Postcoloniality and Feminism. Bloomington: Indi- 